Artigo Original

\title{
Desenvolvimento e aprimoramento de um sistema computacional- Ikapp- de suporte a reabilitação motora
}

\author{
Déborah Marques de Oliveira ${ }^{1}$ \\ Adriana Baltar do Rêgo Maciel ${ }^{1}$ \\ Maíra Izzadora Souza Carneiro ${ }^{1}$ \\ Ana Cláudia de Andrade Cardoso ${ }^{1}$ \\ Alana Elza Fontes da Gama ${ }^{2}$ \\ Thiago de Menezes Chaves ${ }^{2}$ \\ Verônica Teichrieb ${ }^{2}$ \\ Cristiano Coêlho de Araújo ${ }^{2}$ \\ Kátia Karina do Monte-Silva ${ }^{1}$ \\ ${ }^{1}$ Laboratório de Neurociência Aplicada (LANA), Departamento de Fisioterapia, Universidade \\ Federal de Pernambuco, Recife, PE, Brasil \\ ${ }^{2}$ Voxarlabs- Centro de Informática, Universidade Federal de Pernambuco, Recife, PE, Brasil
}

\begin{abstract}
Resumo: A aplicabilidade das Tecnologias Interativas (TIs) na área de saúde, em particular na reabilitação motora, tem sido uma alternativa clínica usada com intuito de estimular maior engajamento do paciente ao seu processo de recuperação que por vezes é extenuante. O presente estudo descreve uma ferramenta tecnológica -lkapp- de suporte a reabilitação motora. Ferramenta essa que busca ampliar as possibilidades dos dispositivos comerciais já existentes no contexto clínico. Sessenta (60) voluntários foram convidados a interagir com as interfaces do setup e do jogo do Ikapp com objetivo de examinar a funcionalidade, grau de aceitação, demandas e limitações para aprimoramentos. Os resultados do presente estudo demonstram altos índices de satisfação pelos participantes. Além disso, os resultados demonstraram que o lkapp é uma ferramenta que agrega valores terapêuticos à ludicidade e motivação de acordo com a perspectiva dos participantes.
\end{abstract}

Palavras-chave: Reabilitação. Interação usuário-computador. Tecnologia.

Development and improvement of a computational system- Ikapp- to support motor rehabilitation

\begin{abstract}
The applicability of Interactive Technologies (ITs) in the health area, especially in motor rehabilitation, has been a therapeutic alternative used aiming to encourage a greater patient engagement in their recovery process that is sometimes lengthy. The present study describes the technological tool (Ikapp) to support motor rehabilitation, which aims to expand the possibility of the commercial devices that is already used in clinical practice. Sixty (60) volunteers were invited to interact with the setup and game interfaces of Ikapp aiming to examine their features, the degree of acceptance, demands and limitations to the enhancement. The results of present study showed high levels of satisfaction for the participants. Furthermore, the results demonstrated that the Ikapp is a tool that adds value to therapeutic playfulness and motivation according to the participant' perspective.
\end{abstract}

Keywords: Rehabilitation. User-computer interface. Technology.

\section{Introdução}

A fisioterapia preconiza um atendimento individualizado, gradativo $e$, frequentemente, extenso. A repetição de exercícios aliada ao grande período de tratamento a que o paciente é submetido e os ganhos diários, geralmente ínfimos, são as principais causas da desmotivação do paciente e sua consequente evasão das clínicas de fisioterapia (MENDONÇA \& GUERRA, 2004). Nesse contexto, o uso de Tecnologias Interativas (Tls) na saúde tem sido uma solução proposta para estimular maior engajamento do paciente ao processo de reabilitação por promover um ambiente de reabilitação mais rico e motivador (KESHNER, 2004; LITTMAN, 1999; SVEISTRUP, 2004). As ferramentas baseada sem Tls visam facilitar a interação entre o usuário (i.e., pacientes) e o computador (TORI, 2005).

A realidade virtual (RV), uma das Tls relevantes da atualidade, tem sido alvo de pesquisas pelo seu uso em programas de reabilitação. A RV proporciona aos pacientes a oportunidade de se engajarem em ambientes virtuais que se assemelham ao mundo real ( $\underline{\mathrm{DE}}$ BRUIN et al., 2010). Diversos estudos propõem a 
$\mathrm{RV}$ como ferramenta de tratamento numa grande variedade de aplicações, da área cognitiva à área motora (DE BRUIN et al., 2010; $\underline{\text { ANTLEY e }}$ SLATER, 2011; LANGE et al., 2010; VIRK e MCCONVILLE, 2006; STANDEN e BROW, 2006). A aplicabilidade da RV na reabilitação motora requer alguns cuidados, tendo em vista que sistemas baseados em RV como o console Wii, por exemplo, foram desenvolvidos com fins de entretenimento. $O$ uso do console Wii pelo paciente pode levá-lo à execução de movimentos com compensações posturais inadequadas. Dessa forma, um acompanhamento contínuo do profissional parece ser imprescindível (SPARKS et al., 2009).

O Ikapp, desenvolvido pelo Centro de Informática em parceria com o departamento de Fisioterapia da Universidade Federal de Pernambuco (UFPE), consiste em um sistema computacional minucioso de rastreamento do movimento corporal (CHAVES et al., 2012; DA GAMA et al., 2012). Este sistema proporciona vantagens para o paciente e para o profissional. Similar aos dispositivos de entretenimento comerciais, o lkapp através de sua interface gráfica também proporciona motivação e desafio aos pacientes. O sistema é de simples manuseio e não necessita de uso de joysticks o que permite que indivíduos com limitações físicas graves sejam capazes de interagir com o dispositivo. Em adição, por sua capacidade de detectar erros de execução de movimentos e de fornecer feedbacks visuais e auditivos para corrigi-los em tempo real, o lkapp permite ao profissional mais autonomia em relação ao acompanhamento contínuo do paciente. Este estudo teve como objetivo descrever o lkapp e examinar - em indivíduos saudáveis - suas funcionalidades, detectando demandas e limitações e, por consequência, prospectando os aprimoramentos que the cabem.

\section{Participantes}

A amostra, selecionada por conveniência, foi composta por sessenta participantes, sendo vinte estudantes do curso de fisioterapia (GF), vinte do curso de computação - ciências e engenharia da computação (GC) e vinte sem formação específica (GSFE), isto é, não vinculados a nenhuma das áreas mencionadas. Os participantes foram recrutados no departamento de Fisioterapia, no Centro de Informática (Cln), bem como no entorno da UFPE. Foram incluídos no estudo indivíduos de ambos os sexos, na faixa etária de 18 a 30 anos e que foram considerados saudáveis, ou seja, aqueles que no momento da inclusão no estudo relataram não possuir qualquer distúrbio neuropsiquiátrico, déficit sensório-motor em membro superior e/ou doença crônica não controlada. Os estudantes de fisioterapia deveriam ter cursado ao menos uma das disciplinas: Cinesiologia e/ou Avaliação em fisioterapia; os de computação, por sua vez, deveriam ter cursado ao menos a disciplina de Jogos virtuais, de Processamento gráfico e/ou de Interface usuário-máquina.

Os critérios de exclusão para participação no estudo foram: ter tido contato prévio com a ferramenta proposta ou ter auxiliado no seu desenvolvimento; possuir qualquer doença congênita e/ou crônica; ser portador de déficit auditivo grave e/ou visual moderado (auto-relato, avaliado através de entrevista) e/ou de déficit cognitivo (escore mínimo de 24, no Mini Exame do Estado Mental-MEEM). O MEEM é um instrumento que avalia a cognição por meio das seguintes funções específicas: orientação temporal e espacial, registro de palavras, atenção, cálculo, linguagem e capacidade construtiva visual. Seu escore pode variar de 0 comprometimento cognitivo grave - a 30 - boa capacidade cognitiva (ALMEIDA, 1998; LOURENCOO; VERA, 2006).

A coleta de dados do presente estudo foi dividida em 2 momentos: $1^{\circ}$ encontro e $2^{\circ}$ encontro. No $1^{\circ}$ encontro, todos os participantes (60) recrutados realizaram as atividades propostas pelo estudo. No $2^{\circ}$ encontro, houve 01 perda no GC e 02 no GSFE, ambas por desistência dos voluntários, restando ao final do estudo cinquenta e sete (57) voluntários.

\section{Material}

Os seguintes equipamentos foram utilizados no presente estudo: um notebook ASUS A42FVX498R Intel Core i5 4GB 750 Gb 14" LCD para execução do jogo, um projetor EPSON® ${ }^{8} 12+$ Powerlite 2800 Lumens para projeção do jogo em uma parede, um sensor Kinect da Microsoft $\AA$ para rastreamento das articulações do corpo e uma câmera digital DSC - W530 da Sony® para registro da interação dos pacientes com a ferramenta (Figura 1). 


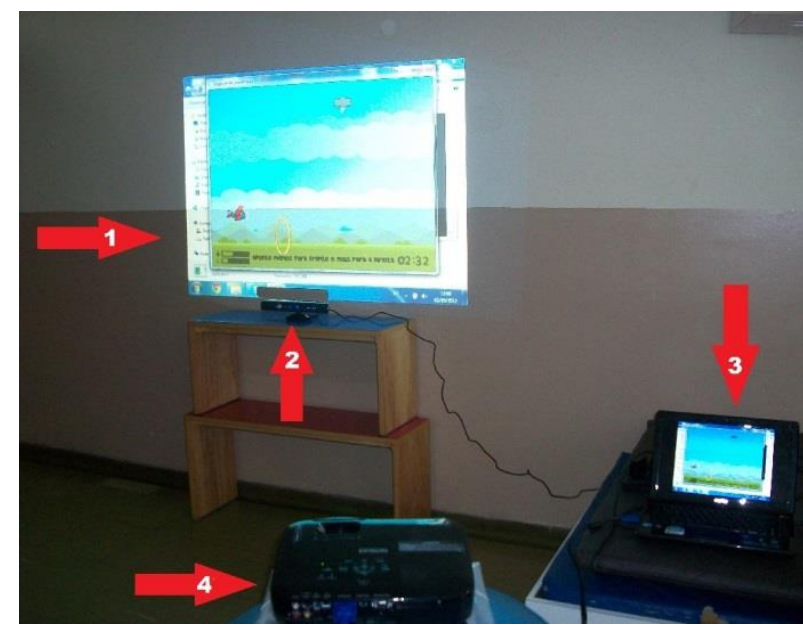

Figura 1. Disposição do ambiente de coletas: (1) projeção do jogo, (2) sensor Kinect, (3) notebook, (4) retroprojetor.

\section{Os seguintes instrumentos e medidas foram} utilizados no estudo:

$\checkmark$ Questionário socioeconômico (QSE): este instrumento foi utilizado para caracterizar a amostra através de dados socioeconômicos e demográficos. O QSE foi elaborado especificamente para $O$ presente estudo baseando-se em questionários que analisaram a aplicabilidade de outras ferramentas tecnológicas ( SUDA et al, 2009). As 13 questões subjetivas que compõem o questionário abordaram aspectos sociodemográficos como renda familiar, nível de escolaridade, meio de transporte utilizado, acesso a meios de comunicação, como televisão e internet, acesso a consoles de vídeo game, entre outros.

$\checkmark$ Questionário de usabilidade e satisfação do jogo (QUS): este instrumento foi desenvolvido com base no estudo de Van Velsen e colaboradores (2007) e examinou a usabilidade e a satisfação dos participantes no contato com o jogo. O questionário aborda questões que foram divididas em domínios, tais como: 1) valor lúdico; 2) bem estar; 3) sensibilidade e 4) valor terapêutico. O QUS possui questões cujas respostas são graduadas de 1 a 5 (muito pouco, pouco, médio, bom e muito bom), bem como questões descritivas. $O$ instrumento foi aplicado após a interação com o jogo no $1^{\circ}$ encontro e $2^{\circ}$ encontro.

$\checkmark$ Questionário de desenvolvimento de interface (QDI): este instrumento teve como base o estudo de Nielsen (1993) e foi elaborado com o objetivo de analisar os modos de definição de tratamento (interface gráfica e bloco de notas). No 1ํ encontro, ao participante do grupo GF foi solicitado escolher o melhor modo, priorizando aspectos como praticidade, layout e nomenclatura ideal para as funcionalidades. Após a escolha pelo participante do melhor modo de definição de tratamento no $2^{\circ}$ encontro, o QDI foi reaplicado com objetivo de avaliar o modo anteriormente escolhido aprimorado. Para quantificar os mesmos aspectos avaliados no teste, O QDI dispõe de escores entre 1 e 5 (muito pouco, pouco, médio, bom e muito bom).

$\checkmark$ Think aloud protocol (TAP): este instrumento foi utilizado com o objetivo de identificar opiniões dos participantes em relação à interação com o setup e com o jogo. Para isso, as pessoas são convidadas a vocalizar seus pensamentos, sentimentos ou opiniões ao interagir com um produto (LEWIS, 1982). De modo a facilitar posterior análise, todos os participantes foram filmados durante as interações com setups e com o jogo.

\section{Procedimentos}

O lkapp é um sistema computacional de suporte a reabilitação motora em que jogos virtuais podem ser controlados através de movimentos, permitindo que 0 fisioterapeuta elabore um plano de tratamento personalizado atentando para as limitações e particularidades de cada usuário. Como representado na Figura 20 sistema lkapp consiste em: (1) módulo de rastreamento corporal, (2) módulo de análise biomecânica, (3) módulo do jogo e (4) módulo de relatório.

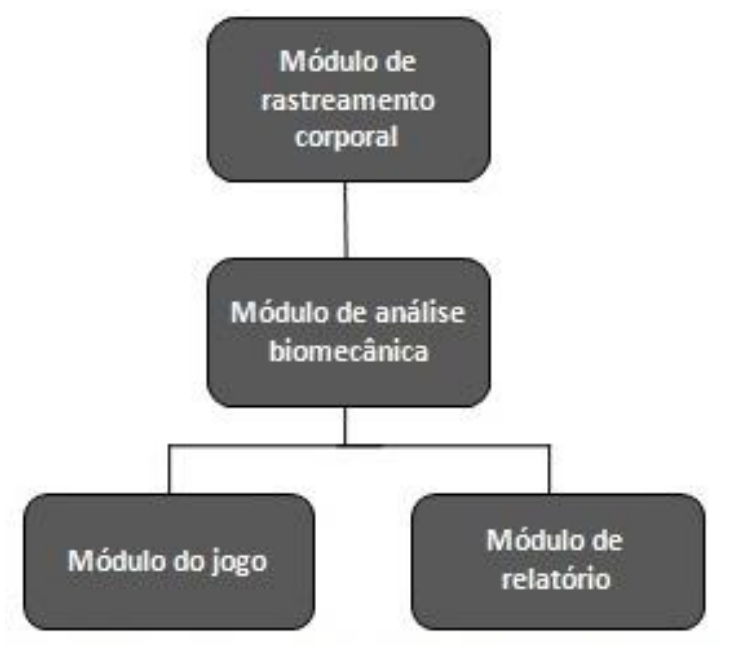

Figura 2. Módulos do sistema Ikapp

O módulo de rastreamento corporal é responsável pelo reconhecimento do corpo humano e registro de dados anatômicos como, por exemplo, a localização das articulações. A fim de permitir tal rastreamento, utiliza-se o sensor 
Kinect da Microsoft@. Já o módulo de análise biomecânica examina os movimentos corporais do ponto de vista biomecânico - usando conceitos de planos, eixos e ângulos - e é capaz de interpretar e avaliar a qualidade do movimento realizado detectando, portanto, compensações posturais. O módulo do jogo é responsável por converter os movimentos corporais registrados no módulo de análise biomecânica em ações no jogo, por exemplo, uma abdução de ombro de um indivíduo no ambiente real equivale a uma elevação de um personagem do jogo no cenário virtual. O módulo de relatório fornece informações sobre o desempenho do usuário durante o uso do sistema como, por exemplo, a angulação máxima atingida durante um movimento corporal e a quantidade de movimentos executados correta ou incorretamente (DA GAMA et al., 2012).

A Figura 3 apresenta o fluxograma de atividades do estudo. Após a formação dos três grupos (GF, GC e GSFE), os participantes foram submetidos a testes de interação com o jogo e, posteriormente, responderam ao QUS. O GF, além de testar o jogo, avaliou os modos de definição de tratamento e respondeu ao QDI. A análise dos questionários foi indispensável para levantar demandas e requisitos para 0 aprimoramento do Ikapp. Quanto ao modo de definição de tratamento foram atendidas as seguintes demandas: a interface ficou mais ilustrativa, tornando a configuração mais intuitiva, dessa forma, diminuindo a chance de erro. Além disso, procurou-se explicar itens necessários para configuração e reduzir a quantidade de informações na tela. Em relação ao jogo houve um aumento considerável na sensibilidade, aprimoramento do cenário gráfico e adição de feedbacks audiovisuais. Na versão aprimorada havia possibilidade de configurar o tempo de manutenção em determinada postura a fim de evitar fadiga. Cerca de um mês após $01^{\circ}$ encontro, os participantes foram novamente convidados a comparecer ao laboratório para realizar um $2^{\circ}$ encontro para teste do jogo e dos modos de definição de tratamento, agora com suas versões aprimoradas. As coletas eram realizadas em um ambiente isolado para evitar dispersão.

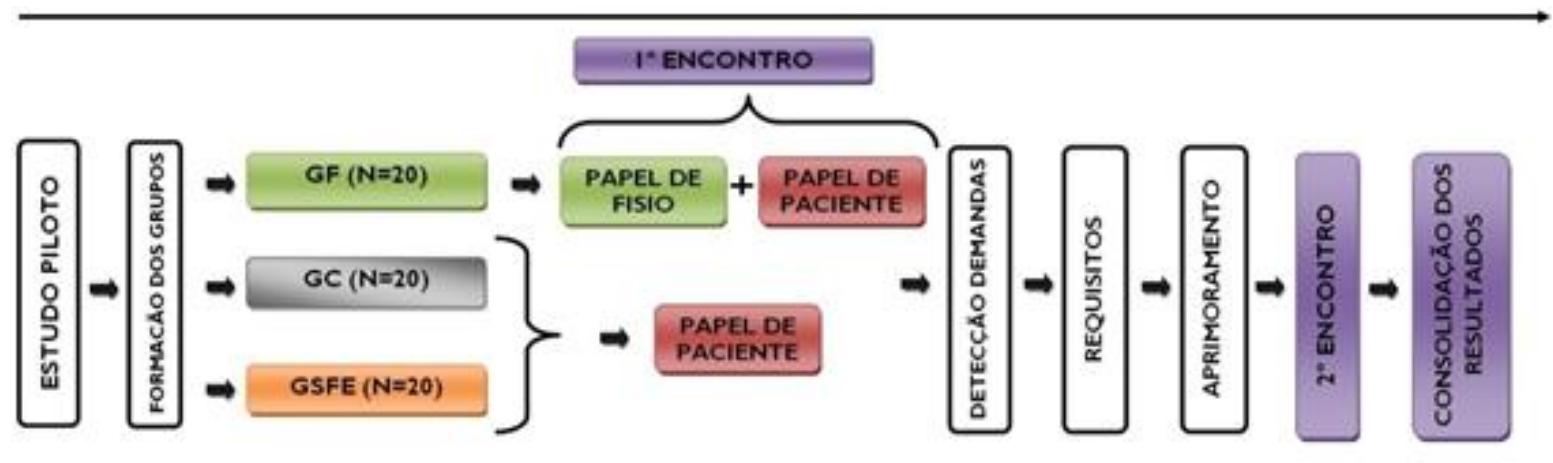

Figura 3. Fluxograma de atividades do estudo

Durante as coletas, após ouvir um áudio instrucional explicando qual atividade seria realizada, os participantes eram posicionados à frente $(\approx 1,5$ metro) da projeção do cenário virtual e solicitados a interagir com o jogo por três minutos. Na versão inicial do Ikapp, o cenário do jogo (Figura 4) era composto por: (i) um golfinho, controlado pelos movimentos do voluntário, abdução e adução do ombro que correspondia, respectivamente, a elevação e depressão do golfinho no ambiente virtual e (ii) por elementos gráficos como moedas, submarinos e peixes. Cada elemento tinha uma representatividade no jogo e exigia uma ação específica dos participantes, o qual através do movimento do golfinho deveria cumprir as seguintes tarefas: (i) colher moedas e (ii) desviar dos peixes e submarinos, dispostos em diferentes alturas. 


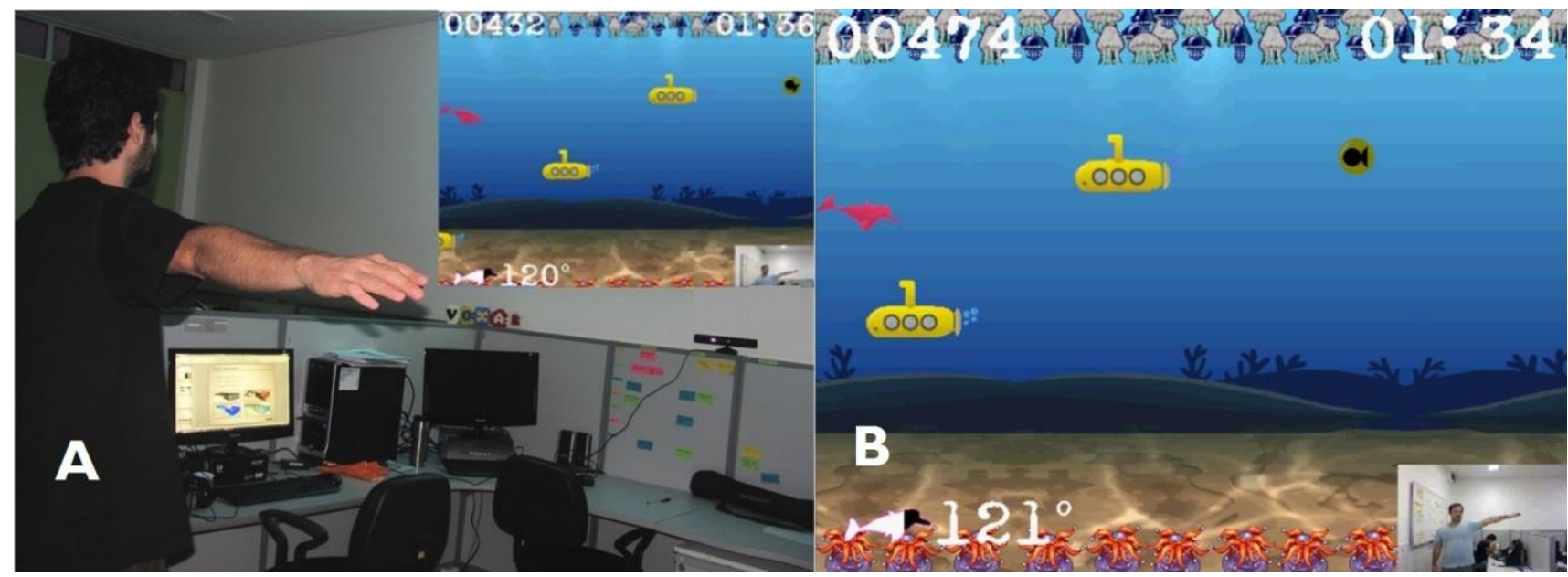

Figura 4. Usuário interagindo com o jogo (A); Cenário do jogo composto por um golfinho e por elementos gráficos, moedas e submarinos $(\mathrm{B})$.

A disposição dos elementos gráficos ao longo do cenário tinha o objetivo de induzir o usuário a realizar contrações isométricas e isotônicas através de movimentos de abdução e adução. Seguidos obstáculos (por exemplo, peixes e submarinos) em uma mesma altura exigiam que 0 usuário permanecesse com o membro superior numa mesma posição, resultando assim, em contração isométrica. Os mesmos obstáculos distribuídos em alturas distintas obrigavam 0 voluntário a realizar abdução/adução do ombro resultando em contrações isotônicas. Durante a interação com o jogo, aos participantes foi solicitado realizar o mesmo padrão de atividade, definido através da disposição de elementos gráficos. Para avaliação do setup foi solicitado ao GF que elaborasse o mesmo plano de tratamento no $1^{\circ}$ encontro e $2^{\circ}$ encontro. Durante o jogo, de modo a alertar o usuário, feedbacks visuais e auditivos (sons) indicavam erros de execução do movimento.

\section{Interação com os setups de definição de tratamento}

Setup ou modo de definição de tratamento consiste em uma ferramenta por meio da qual o fisioterapeuta pode editar os parâmetros do jogo e definir um plano de tratamento específico para cada paciente. Apenas o GF foi submetido a testes com 0 setup de determinação de tratamento, afinal, somente este grupo teria subsídios para explorar o setup, ou seja, elaborar um plano de tratamento. Os parâmetros definidos pelos participantes no setup configuravam a disposição dos elementos gráficos no jogo. Durante o estudo dois tipos de interação com setups foram testados. $\mathrm{N}^{0} 1^{\circ}$ encontro, os indivíduos foram convidados a interagir com dois tipos de setups que funcionavam de maneira distinta, bloco de notas e interface gráfica. Foi solicitado aos participantes que configurassem nos setups sempre a mesma atividade: exercícios de abdução e adução do ombro. $\mathrm{N}^{0} 2^{\circ}$ encontro, os voluntários interagiram apenas com o setup apontado por eles como o mais usual no $1^{\circ}$ encontro. O setup mais usual foi aquele que obteve uma maior pontuação no QUS. Para evitar dispersão do participante e deixá-lo mais atento às instruções sobre a atividade a ser desempenhada, um abafador acústico foi utilizado durante as sessões. Em adição, para eliminar o efeito "aprendizagem prévia" dos voluntários, a ordem de interação com os dois modos de definição do tratamento era sorteada.

\section{Definição de tratamento}

Para definir o tratamento utilizando o setup bloco de notas (Figura 5), os participantes tinham que digitar todos os parâmetros requeridos pelo sistema para executar o jogo, o que na fisioterapia, corresponde a elaboração do plano de tratamento. Os parâmetros eram solicitados através de perguntas objetivas, por exemplo "informe as articulações envolvidas no movimento". 


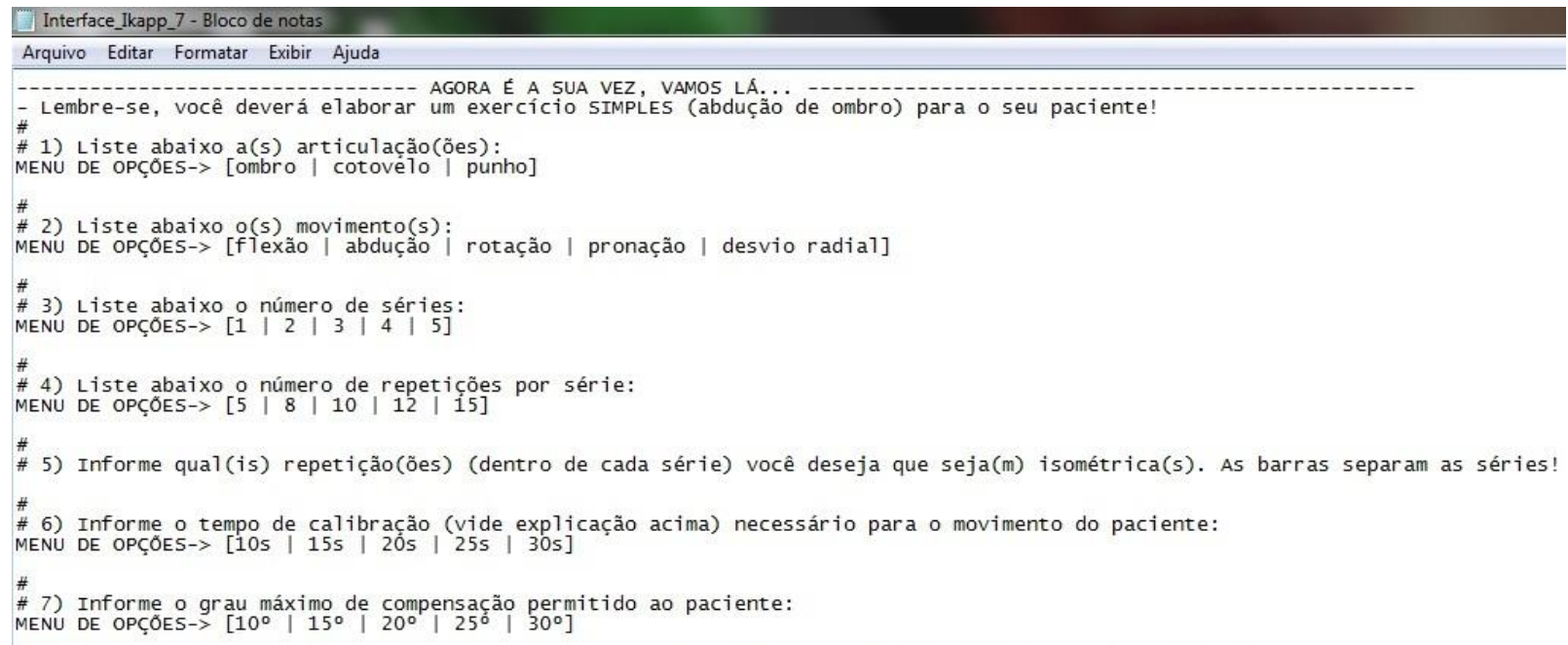

Figura 5. Definição de tratamento usando setup bloco de notas (interface textual).

Para definir o plano de tratamento através do setup interface gráfica, ilustrado na Figura 6 , os participantes deveriam configurar os parâmetros necessários para execução do jogo (elaboração do plano de tratamento) por meio de cliques usando um mouse.

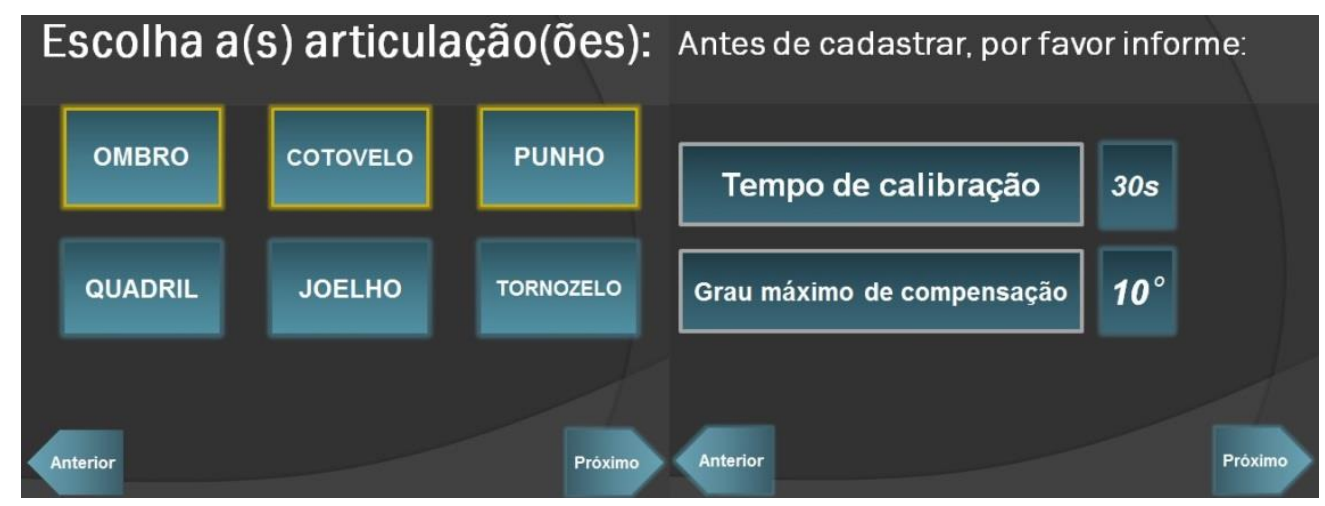

Figura 6. Definição do tratamento usando setup interface gráfica (mouse)

\section{Análise dos dados}

Os dados coletados do QSE foram tabulados em planilha do Microsoft ${ }^{\circledR}$ Excel e submetidos a uma análise descritiva. Medidas de tendência central e de dispersão (média e erro padrão) foram utilizadas para as variáveis quantitativas e medidas de frequência para as variáveis categóricas.

Quanto ao TAP, todos os vídeos das coletas dos voluntários eram analisados por dois examinadores distintos e previamente treinados. As informações foram listadas e os dados considerados relevantes foram expressos na seção de resultados. Em caso de divergência de opiniões, os pesquisadores entraram em consenso.
Os dados obtidos através do QDI no 2o encontro foram analisados sob dois domínios: praticidade (relativo às perguntas sobre facilidade de interação, linguagem e rapidez do setup) e layout (relativo às perguntas sobre possível confusão do usuário ao interagir com a definição do tratamento, poluição visual e chance de erro ao manuseá-lo).

Os dados obtidos através do QUS foram analisados sob quatro domínios: valor lúdico (relativos à diversão, motivação, fisioterapia tradicional x game, cenário e desafio); bem estar (relativo à linguagem, conforto e facilidade); sensibilidade (relativo ao controle do jogo e alta sensibilidade); e valor terapêutico (relativo ao feedback corretivo, incentivos a realizar movimentos corretos, enfoque terapêutico, 
ferramenta complementar, autonomia do terapeuta e visão holística).

Para apresentação dos resultados, os escores do QDI e QUS foram classificados em satisfeitos (escores 4 e 5) e insatisfeitos (1 a 3).

\section{Resultados}

Satisfação do usuário e eleição do modo de definição do tratamento

Com relação ao dominío praticidade, observou-se que quanto à facilidade no manuseio, $90 \%$ dos usuários preferiram o setup gráfico (Mouse), $75 \%$ indicaram que sua linguagem é mais acessível e $75 \%$ afirmam ser mais rápida esta forma de configuração (Figura 7.A).Com relação ao domínio layout, notou-se que $50 \%$ dos usuários ficaram confusos durante a interação com bloco de notas (BN), apontando este setup como mais poluído e com maior chances de erro durante a interação (Figura 7.B).

As demandas e requisitos levantados durante as coletas iniciais permitiram o aprimoramento do setup gráfico e, no $2^{\circ}$ encontro , foi solicitado que - usuário quantificasse sua satisfação com relação a este setup. No domínio praticidade, quanto à facilidade e linguagem, $78,5 \%$ dos usuários afirmaram estar satisfeitos. Com relação a rapidez a satisfação atingiu $85,7 \%$ dos voluntários. Já no domínio layout, altos índices de satisfação foram obtidos nos aspectos poluição $(92,8 \%)$ e chance de erro $(71,4 \%)$. No entanto, observou-se que $50 \%$ dos indivíduos ainda encontravam-se confusos durante a interação(Figura 8).
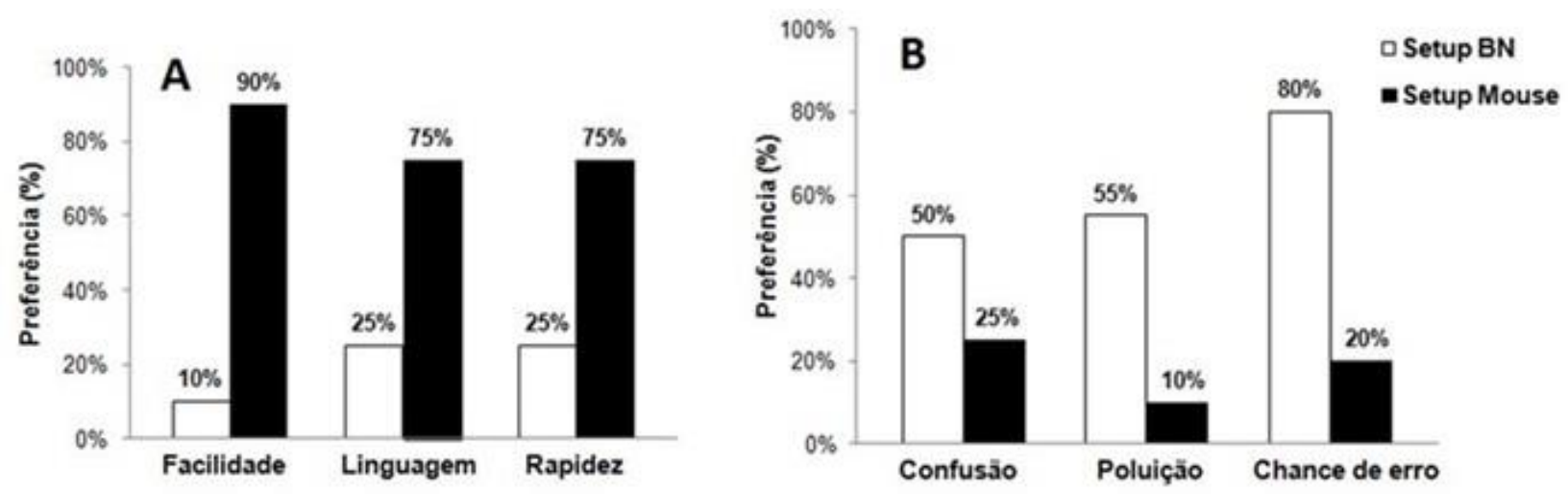

Figura 7. Análise da preferência dos usuários do grupo de fisioterapia (GF) entre os setups de definição do tratamento - mouse e bloco de notas - nos domínios praticidade $(\mathrm{A})$ e layout $(\mathrm{B})$, durante $01^{\circ}$ encontro.

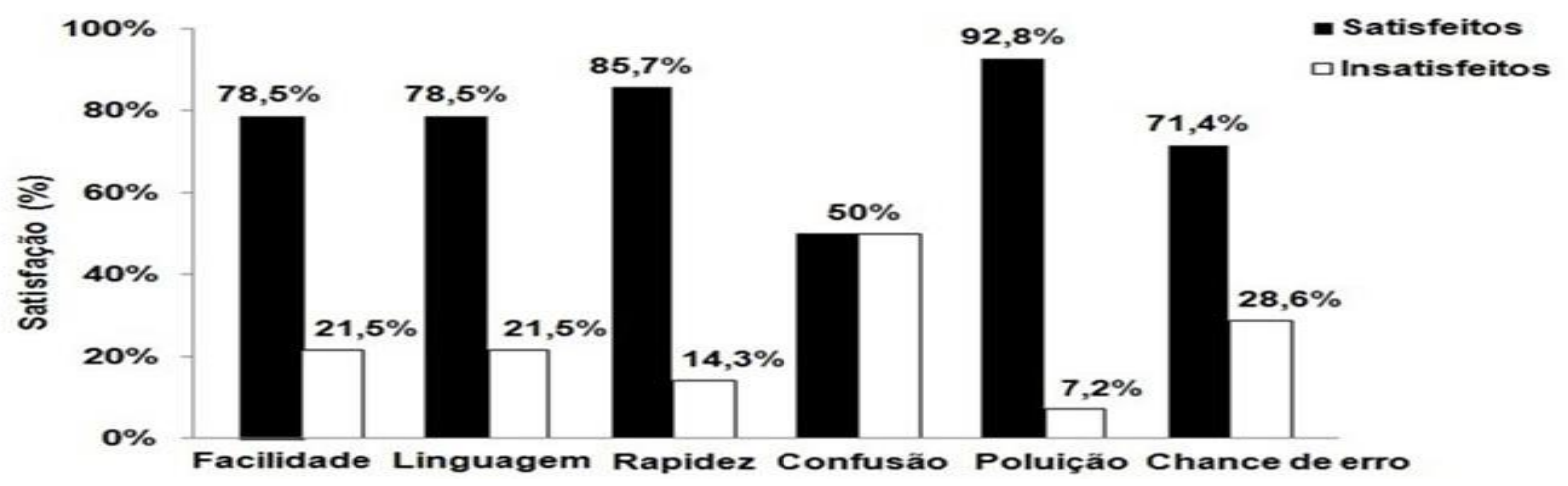

Figura 8. Índice de satisfação dos usuários com a versão aprimorada da interface gráfica de definição do tratamento após atender as demandas levantadas durante $01^{\circ}$ encontro.

\section{Interação com Jogo do Ikapp}

Analisando os dados do QUS no $1^{\circ}$ encontro, com relação ao domínio valor lúdico (Figura 9.A) e bem estar (Figura 9.B), observou-se que a maioria dos participantes dos três grupos (GF, GC e GSFE) afirmaram estar satisfeitos. 

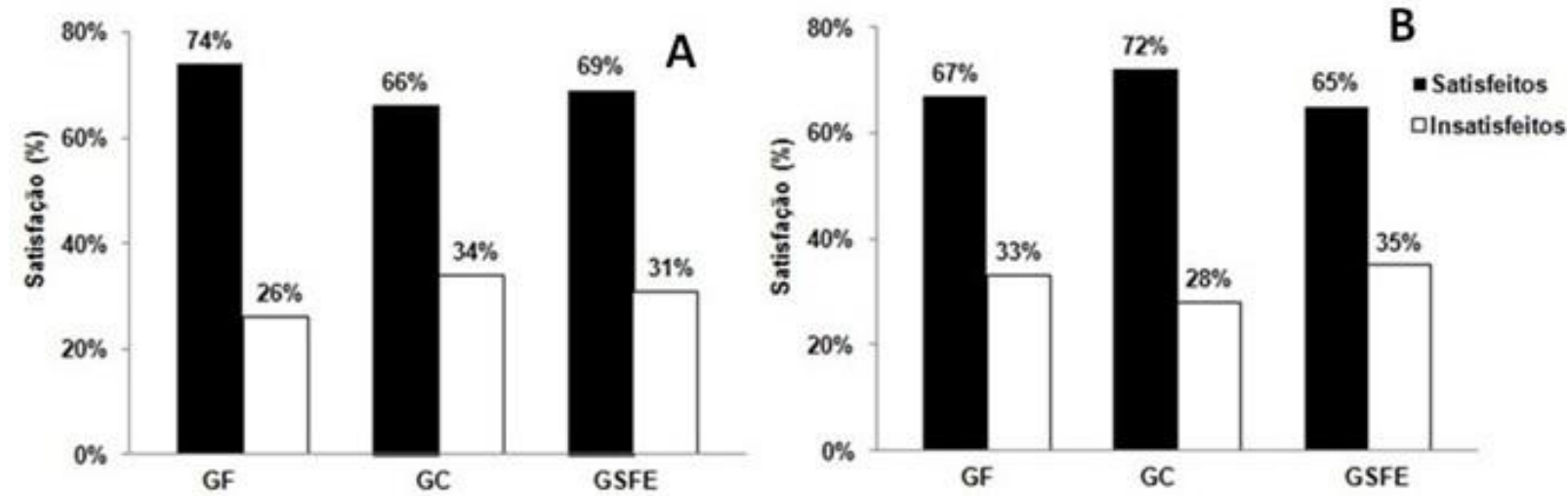

Figura 9. Análise da satisfação (\%) do GF, do GC e do GSFE, durante o $1^{\circ}$ encontro, sobre o domínio valor lúdico $(A)$ e domínio bem estar $(B)$.
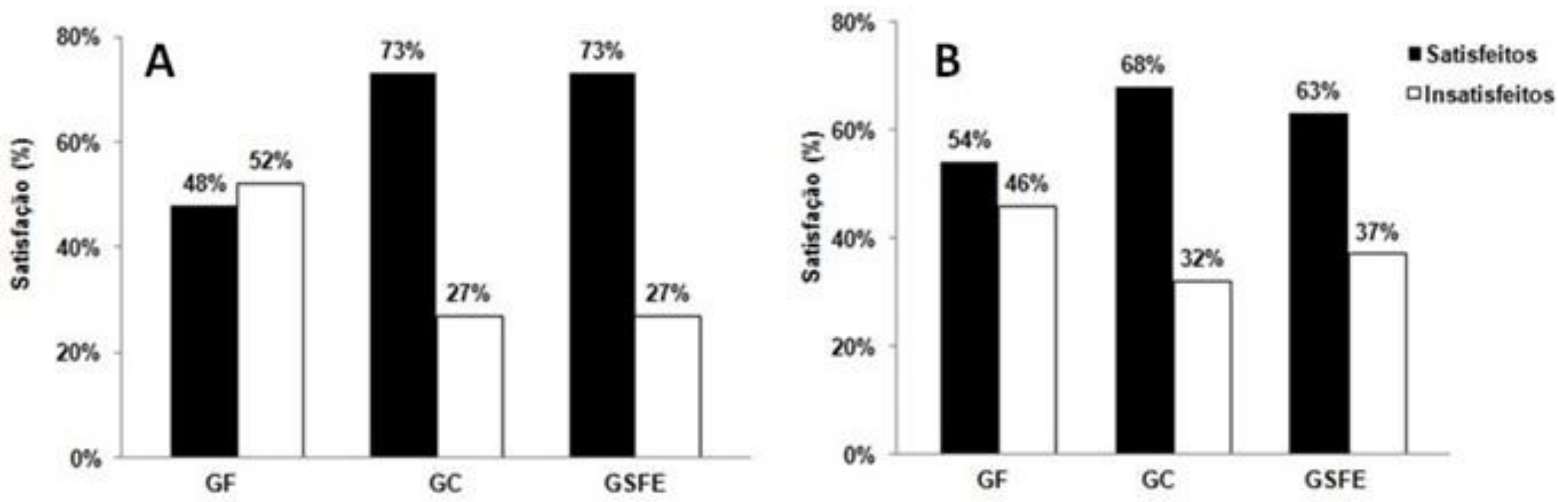

Figura 10. Análise da satisfação (\%) do GF, do GC e do GSFE, durante o $1^{\circ}$ encontro, sobre a sensibilidade (A) e o domínio valor terapêutico (B).

No domínio sensibilidade, evidenciou que apenas no grupo de fisioterapia a maioria dos usuários (52\%) declarou-se insatisfeita (Figura 10.A). O GF também foi o grupo de usuários que menos afirmou estar satisfeito com o dominío valor terapêutico (Figura 10.B).

As demandas e requisitos levantados forneceram subsídios para o aprimoramento do jogo. $\mathrm{Na}$ versão aprimorada, além da sensibilidade que foi consideravelmente aumentada, o cenário do jogo foi modificado. $O$ novo cenário era composto por (i) um avião, controlado pelos movimentos do usuário, e (ii) por elementos gráficos (argolas, balões de gasolina e nuvens). Cada elemento tinha uma representatividade no jogo e exigia uma ação específica dos voluntários, o qual através do movimento do avião deveria cumprir as seguintes tarefas: (i) atravessar as argolas amarelas, (ii) colher os balões de gasolina e (iii) desviar das nuvens, dispostas em diferentes alturas (Figura 11). Como forma de feedback, o avião apresentava-se com contornos de cores diferentes, verde quando o movimento era realizado corretamente e vermelho quando havia erros de execução. Em adição, um feedback auditivo era emitido a fim de corrigir as compensações posturais que o usuário poderia estar cometendo.

\section{Avaliação da satisfação dos usuários com o jogo aprimorado}

No $2^{\text {o }}$ encontro foi solicitado que os participantes de todos os grupos interagissem com a versão aprimorada do jogo. Os dados coletados neste momento são demonstrados de forma comparativa com os obtidos no $1^{\circ}$ encontro de modo a evidenciar a melhora dos aspectos examinados.

Com relação ao valor lúdico (Figura 12.A), a satisfação ascendeu em 18,8 \% para o GF, 7,7\% para o GC e $17,6 \%$ no GSFE. Um aumento mais acentuado foi verificado com relação ao domínio bem estar (Figura 12.B), sendo o crescimento de $31 \%$ na satisfação do GF e GSFE e $15,5 \%$ do GC. 


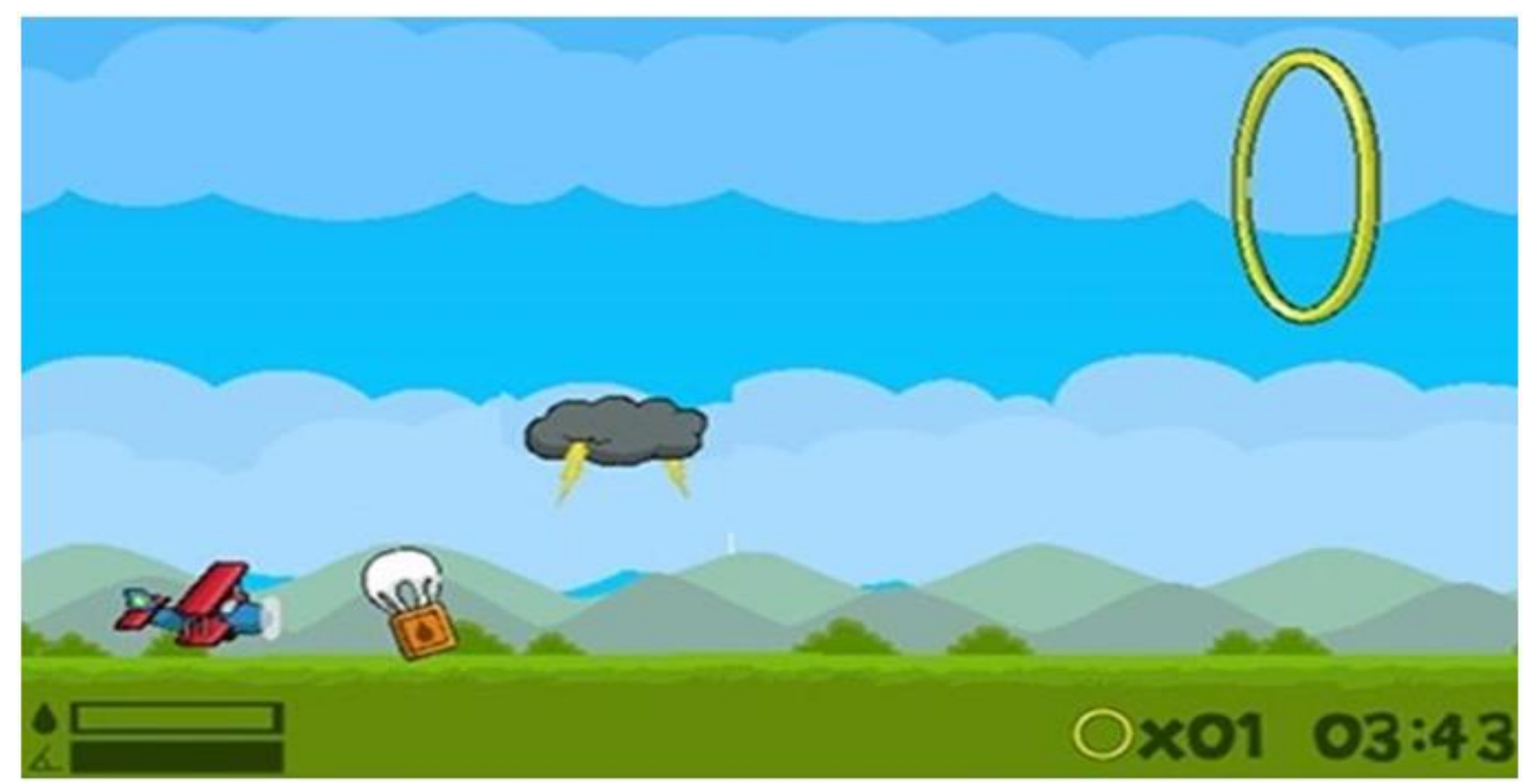

Figura 11. Interface aprimorada do jogo (avião) com os elementos gráficos dispostos pelo cenário como argolas, nuvens e balões de gasolina.
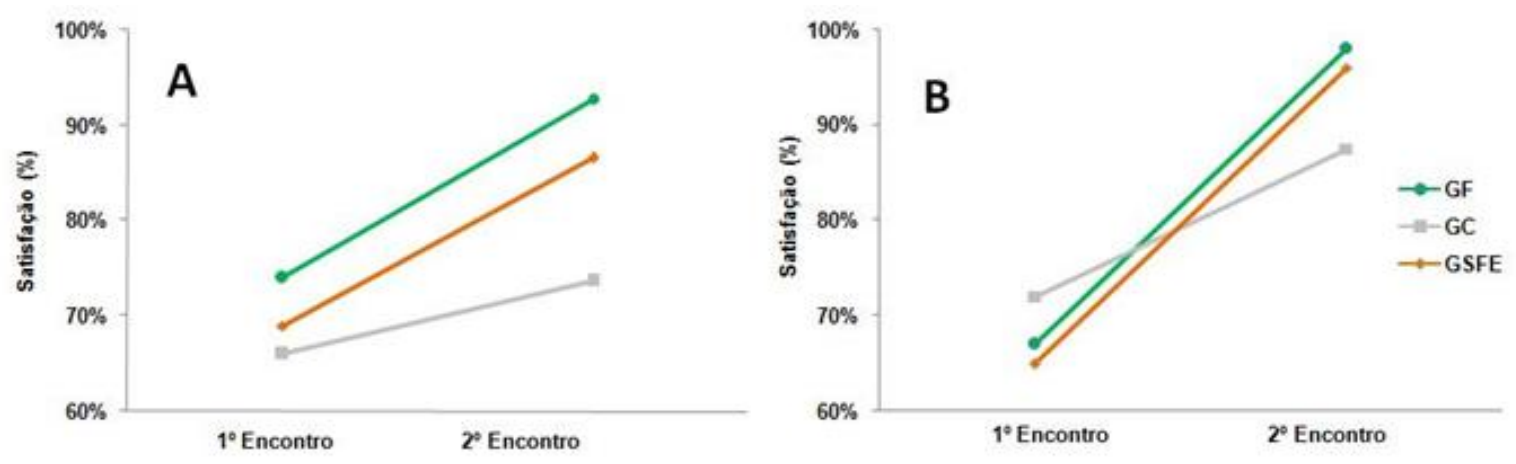

Figura 12. Índice de satisfação (\%) do GF, GC e GSFE sobre o domínio valor lúdico (A) e bem estar (B) no $1^{\circ}$ encontro e $2^{\circ}$ encontro, ou seja, antes e após o aprimoramento respectivamente.
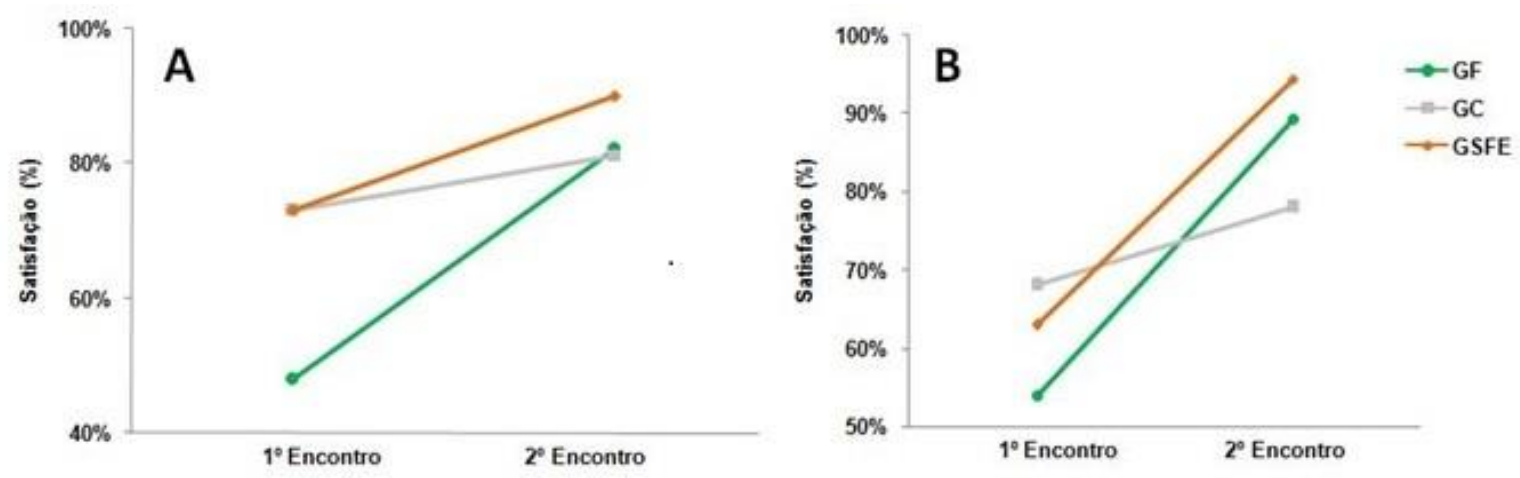

Figura 13. Índice de satisfação (\%) do GF, GC e GSFE sobre o domínio sensibilidade (A) e valor terapêutico (B) no $1^{\circ}$ encontro e $2^{\circ}$ encontro, ou seja, antes e após o aprimoramento respectivamente. 


\section{Discussão}

Após o aprimoramento, os resultados do presente estudo evidenciaram que o lkapp é uma ferramenta tecnológica que agrega valores terapêuticos à ludicidade e motivação de acordo com a perspectiva do usuário.

Para atender ao seu objetivo que é ser uma ferramenta que atinja aos dois tipos de usuários (fisioterapeutas e pacientes), o lkapp é constituído por dois componentes: (i) definição de tratamento, voltado para o usuário fisioterapeuta e (ii) interface do jogo, voltada para o usuário paciente. Cada componente envolve aspectos fundamentais para que o dispositivo possa ser aplicado no contexto terapêutico. Estes aspectos foram testados e aprimorados no presente estudo, sob a perspectiva dos usuários, e fazem parte das etapas que antecedem a implementação de novos dispositivos tecnológicos nos serviços de saúde.

Segundo Souza e colaboradores (1999), a avaliação da interface é de suma importância para $o$ seu processo de desenvolvimento $e$ aprimoramento, já que, por meio dela, é possível estimar o sucesso ou insucesso da aplicabilidade das suas funcionalidades e da interação do usuário com este componente do sistema proposto. Ainda neste contexto, sabe-se que a avaliação de usabilidade segundo Scholtz (1995) é um processo capaz de detectar um "gap" entre o que realmente é o sistema e a ideia que o usuário tem acerca dele. Usabilidade é compreendida como o termo técnico que descreve a qualidade do uso de uma interface (BEVAN, 1995).

Com relação à avaliação da interface de definição do tratamento realizada pelos usuários do GF, o setup gráfico foi indicado como o melhor após $01^{\circ}$ encontro, comparado com o de bloco de notas. Este resultado está em concordância com Bao e colaboradores (2006) que afirmaram que quanto menos ação de clicar e/ou digitar pelos usuários, mais eficaz será a interação deles com este componente. Fato evidenciado também no $2^{\circ}$ encontro do presente estudo através dos altos índices de satisfação dos usuários nos itens facilidade, rapidez (domínio praticidade) e baixa chance de erro (domínio layout), resultado que evidencia a alta usabilidade deste setup. De fato, estudos prévios demonstram que a qualidade da usabilidade está associada aos aspectos facilidade, rapidez e baixa chance de erro (NIELSEN, 1993).

Um dos aspectos avaliados (confusão) não aponta para essa direção de alta usabilidade do setup de definição do tratamento, uma vez que apenas metade dos usuários declarou-se satisfeita com a interface aprimorada. Este resultado, porém, foi considerado contraditório, já que todos os outros aspectos foram avaliados positivamente. Para o setup ser usual, segundo Baerentsen (2000), ele deve ser o mais intuitivo possível, ou seja, simples e fácil o suficiente para que o usuário possa manuseá-lo sem nenhuma explicação prévia. Diante dos resultados obtidos, é possível que a versão aprimorada do setup de definição de tratamento ainda não seja a ideal para os usuários.

Com a interface do jogo, o lkapp se propõe a resolver problemas apontados na prática tradicional da fisioterapia, tais como monotonia, necessidade de um acompanhamento contínuo do fisioterapeuta, evasão do tratamento, inviabilidade de reavaliações diárias do paciente, entre outros. O Ikapp poderá no futuro beneficiar pacientes com distúrbios neurológicos, lesões traumáticas e patologias reumatológicas.

Com relação à prática tradicional da fisioterapia, estudiosos apontam que o caráter repetitivo, extenso e monótono dos exercícios repercute, muitas vezes, em desestímulo e abandono da terapia (MENDONCCA e GUERRA, 2004). Observando os resultados da avaliação do "valor lúdico do jogo" e "bem estar", conclui-se que o lkapp resolve esta problemática, haja vista os altos índices de satisfação de todos os grupos de usuários avaliados no quesito motivação, definida com uma sensação subjetiva de envolvimento com a ferramenta. Assim, quanto maior a motivação com as atividades terapêuticas, maior o engajamento do usuário com 0 seu processo de reabilitação, consequentemente, menor a chance de abandono do tratamento. De fato, estudos apontam que uma das principais razões para o uso das Tls na reabilitação é que ambiente lúdico proporcionado pela interatividade e jogos, tais como na RV, potencializa o grau de envolvimento do paciente com o tratamento (RIZZO e KIM, 2005). Diferente das outras ferramentas tecnológicas de interação, - Ikapp permite que o indivíduo continue interagindo com o sistema com fins terapêuticos sem necessariamente ser supervisionado 
constantemente pelo terapeuta. A presença do fisioterapeuta é essencial para evitar o risco de lesão com uso dos dispositivos interativos comerciais. Este fato foi evidenciado por Sparks e colaboradores (2009) ao verificar altos índices de lesões associadas ao uso indiscriminado, sem acompanhamento, destes dispositivos. A existência de feedbacks corretivos no Ikapp garante, diferente dos outros dispositivos, que o paciente possa ficar um tempo sem o suporte direto do fisioterapeuta, evitando compensações posturais e possíveis erros de execução de movimento. De fato, os altos índices de satisfação sobre o valor terapêutico pelo GF, evidenciam que o Ikapp fornece feedbacks suficientes, em tempo real, e isto como sugerido por Kesnher (2004) pode evitar alguns tipos de lesão nestes usuários.

Outra limitação dos dispositivos interativos comerciais deve-se ao fato de que a maioria das ferramentas necessita que o usuário faça uso de dispositivos auxiliares para controlar o jogo. No entanto, o Ikapp, através da captação de imagem, soluciona esta lacuna. Neste estudo, os altos índices no domínio sensibilidade corroboram com a ideia de que os usuários tinham liberdade de movimento que não requeria uso de nenhum dispositivo auxiliar e também afirmaram ter alto controle do personagem (golfinho ou avião) durante o jogo.

Por fim, pode-se concluir que o lkapp mostrouse uma ferramenta eficaz, sendo capaz de aliar ludicidade e motivação ao processo de fisioterapia. Desta forma, acredita-se que o uso de dispositivos pode contribuir para atenuar os altos índices de evasão na prática clínica. A versão atual do Ikapp pode promover acessibilidade, de modo que, boa parte dos indivíduos portadores de limitações (necessidades especiais) sinta-se entusiasmada a realizar sua rotina de exercícios com um videogame. Nota-se, no entanto, a necessidade de continuidade do presente estudo, testando a usabilidade/satisfação do Ikapp sob a perspectiva do paciente com reais necessidades de reabilitação motora e, por fim, um estudo (ensaio clínico) que evidencie o valor terapêutico do lkapp comparando-o com outro dispositivo interativo.

\section{Referências}

ALMEIDA, O.P. Mini exame do estado mental e o diagnóstico de demência no Brasil. Arquivos de Neuro-psiquiatria, São Paulo, v.56, n.3, p. 605612, 1998.
ANTLEY, A.; SLATER, M. The effect on lower spine muscle activation of walking on a narrow beam in virtual reality. IEEE Transactions on Visualization and Computer Graphics, v.17, n.2, p.255-259, 2011. DOI:

http://dx.doi.org/10.1109/TVCG.2010.26

BAERENTSEN, K.B. Intuitive user interfaces. Scandinavian Journal of Information Systems, v.12, n.1, p.29-60, 2000. Disponível em: $<$ http://aisel.aisnet.org/sjis/vol12/iss1/4>. Acesso em: maio de 2012.

BAO, X.; HERLOCKER, J.L.; DIETTERICH, T.G. Fewer clicks and less frustration: reducing the cost of reaching the right folder. $\mathbf{1 1}^{\text {th }}$ international conference on intelligent user interfaces,Sydney, p.178-185, 2006.

BEVAN, N. Usability is quality of use. Advances in Human Factors/Ergonomics, v.20, n.6, p.349354, 1995. DOI: http://dx.doi.org/10.1016/S09212647(06)80241-8

CHAVES, T.M. et al. Human Body Motion and Gestures Recognition Based on Checkpoints. In: XIV SYMPOSIUM ON VIRTUAL AND AUGMENTED REALITY, Niterói: IEEE Proceedings of XIV Symposium on Virtual and Augmented Reality, 2012.

DA GAMA, A. E. F.et al. Ikapp a rehabilitation support system using kinect. In: XIV SYMPOSIUM ON VIRTUAL AND AUGMENTED REALITY, Niterói: Proceedings of XIV Symposium on Virtual and Augmented Reality, 2012.

DE BRUIN, E.D. et al.Use of virtual reality technique for the training of motor control in the elderly. Some theoretical considerations.

Zeitschrift fur Gerontologie und Geriatrie, Darmstadt, v.43, n.4, p. 229-234, 2010.

KESHNER, E.A. Virtual reality and physical rehabilitation: a new toy or a new research and rehabilitation tool? Journal of Neuroengineering and Rehabilitation, London, v.1, n.1, 2004.

MENDONÇA, K.M.P.P.; GUERRA, R.O. Desenvolvimento e validação de um instrumento de medida da satisfação do paciente com a fisioterapia. Revista Brasileira de Fisioterapia, São Carlos, v.11, n.5, p.369-376, 2007.

LANGE, B. et al.Development of an interactive game-based rehabilitation tool for dynamic balance training.Topics in Stroke Rehabilitation, Frederick, v.17, n.5, p.342 -352, 2010.

LEWIS, C. Using the" thinking-aloud" method in cognitive interface design. IBM Thomas J.

Watson Research Center, 1982. 
LITTMAN, M. Enhancing instruction through virtual reality. Proceedings of International Conference on Tissue Engineering, p.31-33, 1999.

LOURENÇO,R.A.; VERAS, R.P. Mini-Exame do Estado Mental: características psicométricas em idosos ambulatoriais. Revista de Saúde Pública, São Paulo, v.40, n.4, p. 712 718, 2006.

NIELSEN, J. Usability engineering, San Francisco: Morgan Kaufmann, 1993

RIZZO, A.S.; KIM, G.J. A SWOT analysis of the field of virtual reality rehabilitation and therapy.

Presence: Teleoperators \& Virtual

Environments, Massachusetts, v.14, n.2, p.119146, 2005.

SCHOLTZ, J.Usability: what's it all about? Software Quality Journal, New York, v.4, n.2,p.95-100, 1995.

SOUZA, C.S. et al. Projeto de Interfaces de Usuário: perspectivas cognitivas e semióticas.

Apostila do Curso de Projeto de Interfaces de Usuário Departamento de Informática, PUCRio. Rio de Janeiro-RJ, 1999.

SPARKS, D.; CHASE, D.; COUGHLIN,L. Wii have a problem: a review of self reported Wii related injuries. Informatics in primary care, Abingdon, v.17, n.1, p.55-57, 2009.

STANDEN, P.; BROWN, D. Virtual reality and its role in removing the barriers that turn cognitive impairments into intellectual disability. Virtual

Reality, London, v.10,n.3, p. 241-252, 2006. DOI: http://dx.doi.org/10.1007/s10055-006-0042-6

SUDA, E.Y.; UEMURA,M.D.; VELASCO, E.Avaliação da satisfação dos pacientes atendidos em uma clínica-escola de Fisioterapia de Santo André, SP.Fisioterapia e Pesquisa, São Paulo, p.126-131, 2009.

SVEISTRUP, $\mathrm{H}$. Motor rehabilitation using virtual reality. Journal of Neuroengineering and Rehabilitation, London, v.1, n.2, 2004.

TORI, L. A presença das tecnologias interativas na educação. Revista de Tecnologia e Educação-PUC, São Paulo, v.2, n.1, p.4-16, 2005.

VAN VELSEN, L.; VAN DER GEEST, T; KLAASSEN, R. Testing the usability of a personalized system: comparing the use of interviews, questionnaires and thinking-aloud. In: INTERNATIONAL PROFESSIONAL

COMUNICATION CONFERENCE, Seattle: IEEE

Proceedings of International Professional

Communication Conference, 2007.
VIRK, S.; MCCONCILLE, K.M.V. Virtual reality applications in improving postural control and minimizing falls. In: $28^{\text {th }}$ ANNUAL

INTERNATIONAL CONFERENCE OF THE IEEE ENGINEERING IN MEDICINE AND BIOLOGY SOCIETY, New York: IEEE Proceedings of $\mathbf{2 8}^{\text {th }}$ Annual International Conference of the IEEE Engineering in Medicine and Biology Society, 2006.

Agradecimento: Apoio financeiro da Fundação de Amparo á Ciência e Tecnologia do estado de Pernambuco (FACEPE; $n^{\circ}$ processo: APQ-08681.03/10).

\section{Endereço:}

Kátia Monte-Silva

UFPE - Departamento de Fisioterapia

Av. Jornalista Aníbal Fernandes, s/n,

Cidade Universitária

Recife PE Brasil

50740-560

Telefone: (81) 2126-7579

e-mail:monte.silvakk@gmail.com

Recebido em: 28 de junho de 2012.

Aceito em: 03 de abril de 2013.

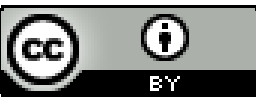

Motriz. Revista de Educação Física. UNESP, Rio Claro, SP, Brasil - elSSN: 1980-6574 - está licenciada sob CreativeCommons - Atribuição 3.0 